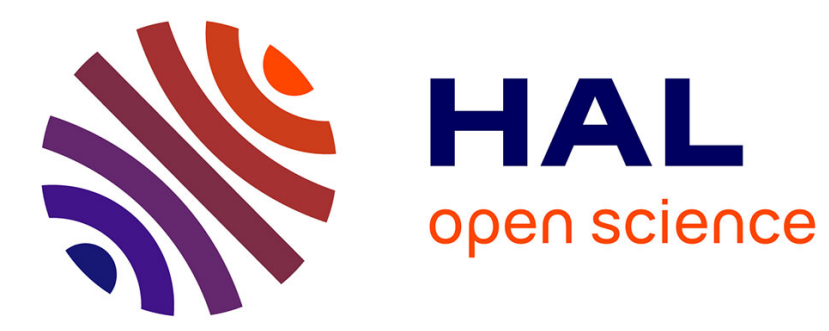

\title{
Psychophysical study of LCD motion-blur perception
}

\author{
Sylvain Tourancheau, Patrick Le Callet, Kjell Brunnström, Borje Andrén
}

\section{To cite this version:}

Sylvain Tourancheau, Patrick Le Callet, Kjell Brunnström, Borje Andrén. Psychophysical study of LCD motion-blur perception. Human Vision and Electronic Imaging XIV., Jan 2009, San Jose, CA, United States. pp.7240-51. hal-00360273

\section{HAL Id: hal-00360273 \\ https://hal.science/hal-00360273}

Submitted on 10 Feb 2009

HAL is a multi-disciplinary open access archive for the deposit and dissemination of scientific research documents, whether they are published or not. The documents may come from teaching and research institutions in France or abroad, or from public or private research centers.
L'archive ouverte pluridisciplinaire HAL, est destinée au dépôt et à la diffusion de documents scientifiques de niveau recherche, publiés ou non, émanant des établissements d'enseignement et de recherche français ou étrangers, des laboratoires publics ou privés. 


\title{
Psychophysical study of LCD motion-blur perception
}

\author{
Sylvain Tourancheau ${ }^{a}$, Patrick Le Callet ${ }^{a}$, Kjell Brunnström ${ }^{b}$, and Börje Andrén ${ }^{b}$ \\ ${ }^{a}$ IRCCyN, University of Nantes \\ ${ }^{b}$ Video and Display Quality, Photonics Dep. \\ Polytech'Nantes, rue Christian Pauc \\ Electrum 236, Acreo AB \\ 44306 Nantes, France \\ tel: +33240683065 \\ sylvain.tourancheau@univ-nantes.fr \\ SE-16440 Kista, Sweden \\ tel: +4686327732 \\ kjell.brunnstrom@acreo.se
}

\begin{abstract}
Motion-blur is still an important issue on liquid crystal displays (LCD). In the last years, efforts have been done in the characterization and the measurement of this artifact. These methods permit to picture the blurred profile of a moving edge, according to the scrolling speed and to the gray-to-gray transition considered. However, other aspects should be taken in account in order to understand the way LCD motion-blur is perceived.

In the last years, a couple of works have adressed the problem of LCD motion-blur perception, but only few speeds and transitions have been tested. In this paper, we have explored motion-blur perception over 20 gray-to-gray transitions and several scrolling speeds. Moreover, we have used three different displays, to explore the influence of the luminance range as well as the blur shape on the motion-blur perception.

A blur matching experiment has been set up to obtain the relation between objective measurements and perception. In this experiment, observers must adjust a stationary test blur (simulated from measurements) until it matches their perception of the blur occuring on a moving edge. Result shows that the adjusted perceived blur is always lower than the objective measured blur. This effect is greater for low contrast edges than for high contrast edges. This could be related to the motion sharpening phenomenon.
\end{abstract}

\section{INTRODUCTION}

Though LCD (liquid crystal display) has become the market leader of monitors and televisions in the last years, motion-blur is still an important issue on these type of displays. This artefact is due to the sample-and-hold behaviour of LCD: the light intensity is held on the screen for the duration of the frame (LC displays are so called hold-type displays). This is particularly annoying when observers are tracking a moving object on LCD: the object is held stationary on the screen during the frame period while observers'eyes are still moving slightly (smooth pursuit). The edges of the object are then displaced on the retina resulting in a blur. ${ }^{1}$

In the last years, efforts have been done in the characterization and the measurement of this artifact. One common measurement system used to characterize LCD motion-blur is referred as MPRT measurement system (for Moving Picture Response Time). ${ }^{2}$ It consists in a high-speed camera tracking a moving edge in order to simulate the smooth pursuit perform by the eyes. Several configurations exists (moving camera on a rail, rotating mirror, etc.) as well as alternative procedures such as the usage of a stationary high-speed camera combined with motion compensation..$^{3,4}$

Moreover, analysis of LCD motion-blur formation blur has shown that the blurred profile of a moving edge can be obtained from the temporal step response of the LCD pixels. ${ }^{5,6}$ As temporal step response measurements are easy to carry out and can be done rapidly for a lot of gray-to-gray transitions, such a method is very useful. A comparison between this analytic method based on temporal response and some camera measurements has been performed for a large number of gray-to-gray transitions, on five recent monitors. ${ }^{7}$ The study has shown that both set of results were very close, even for displays with motion-blur reduction systems such as backlight flashing.

These objective physical measurements permits to know the edge profile that reaches the retina (if the effects of the optics of the eye are neglected). However, some process occur in the retina and further in the visual cortex, 
and one can assume that the perception of the edge will be different from physical measurements. Only few works have been done previously to explore the perception of LCD motion-blur. Someya ${ }^{8}$ performed some blur matching experiment between a moving edge on LCD and a moving edge on CRT. Forty-two transitions have been tested but with only one velocity. A certain accordance between subjective and measured data has been demonstrated, but the complexity of the experimental setup, and particularly the physical differences between LCD and CRT, does not permit to draw some straightforward conclusions. Teunissen et al. ${ }^{9}$ have also developed a blur matching experiment in which the speed of a moving edge was adjusted by observers until the motionblur induced by the speed matches the blur of stationary blurred edge simulated from measurements. Eight transitions and four velocities have been tested, and one display. A very good match has been reported between speeds adjusted by the observers and speeds used to simulate the stationary blurred edge. Finally, Feng ${ }^{10}$ has performed the same type of experiments with one transition and four velocities. Observers were asked to adjust a simulated stationary blurred edge until it matches their perception of a moving edge. A good correlation between subjective results and measurements has been reported, and moving edge was perceived sharper than predicted by measurements.

Those previous results only cover a limited number of transitions, few velocities and one display each. Moreover, some contradictions exists between results of Teunissen et al. and those of Feng, maybe due to the difference of procedures (speed adjustment versus blur adjustment). The goal of this paper is to explore the perception of blur on a moving edge for different gray levels and scrolling speeds with a blur matching procedure, and to repeat experiment on a couple of displays. The relation between psychophysical results and measurements would permit to determine the actual perception of motion-blur by human observers.

\section{PHYSICAL MEASUREMENTS}

In these study, the physical measurement method is based on the temporal step-response, because it presents a higher sample-rate than the camera method, and is easier to carry out and less time-consuming. Moreover, it is easily reproducible from one lab to another and this was an important condition for us since our experiments have been led in two different lab.

Since this analytic method has been widely described in some previous works,${ }^{4-6}$ details are not provided here. The blurred profile of a moving edge is obtained by a convolution of the temporal step-response with a unit one-frame-period wide window. The blurred edge profile is obtained in the temporal domain and the blurred edge time $(B E T)$ is measured on it between $10 \%$ and $90 \%$ of the edge dynamic (cf. Figure 1). As a result, a value of $B E T$ (in temporal units) is obtained for each gray-to-gray transition.

$B E T$ is independent of the speed but the actual blur width (in spatial units) will depend on it. It has been shown from measurements as well as from analysis that, for a given gray-to-gray transition $N_{i} \rightarrow N_{j}$, the blurred edge width $(B E W)$ varies linearly with the velocity of the moving edge $V$ with a factor equal to $B E T$. Here, $B E W_{m}$ denotes the measured blurred edge width:

$$
B E W_{m}=B E T \cdot V
$$

In the following, $V$ is expressed in pixels per frame, $B E W_{m}$ in pixels and $B E T$ in frames.

\section{PSYCHOPHYSICAL EXPERIMENT}

\subsection{Description}

Psychophysical experiment has been conducted according to a procedure that is very similar to that used by Feng: ${ }^{10}$ observers have to adjust the blur of a stationary edge until it matches their perception of the motion-blur occurring on a moving edge. The test field is split into two parts as illustrated in Figure 2a. The stationary 


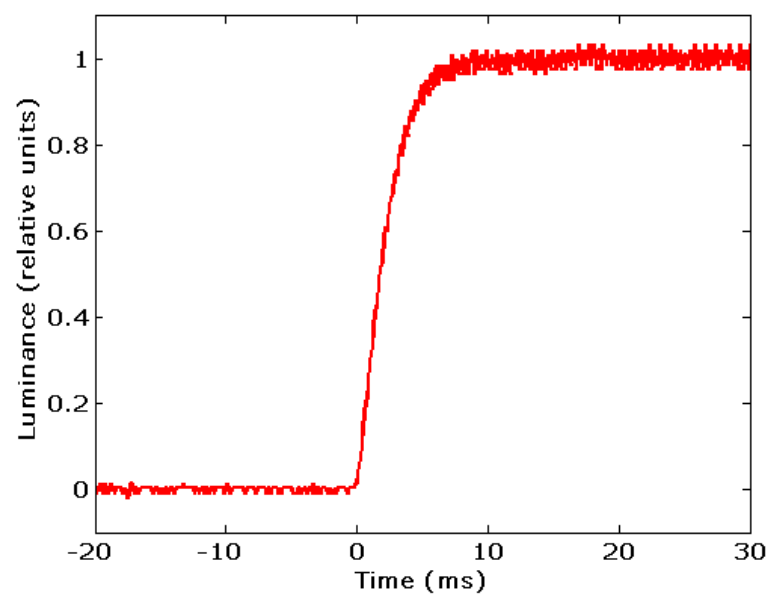

(a) Temporal step-response.



(b) Blurred edge profile.

Figure 1. Blurred edge profile and blurred edge time BET (b) obtained from the temporal step-response of the considered transition (a) (see text).

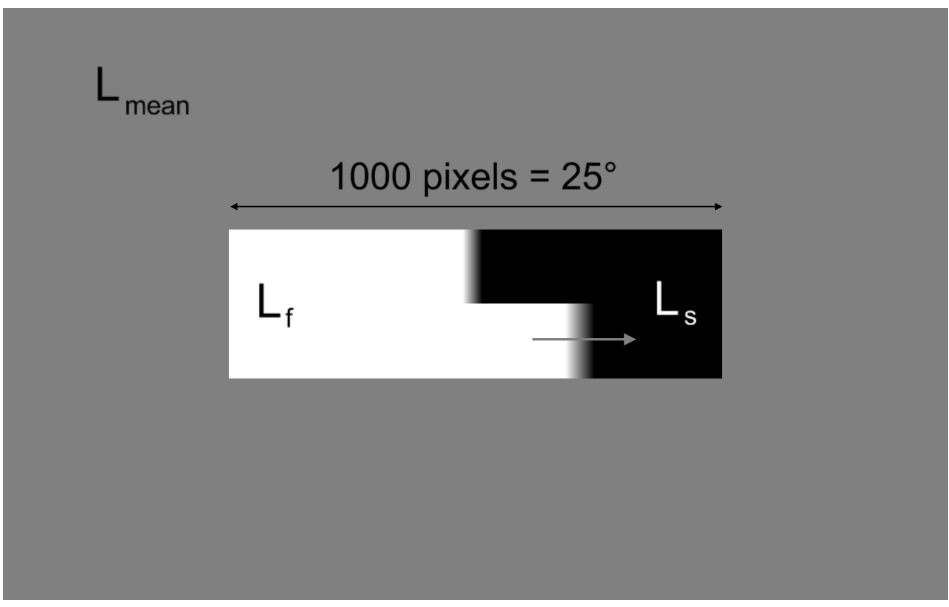

(a)

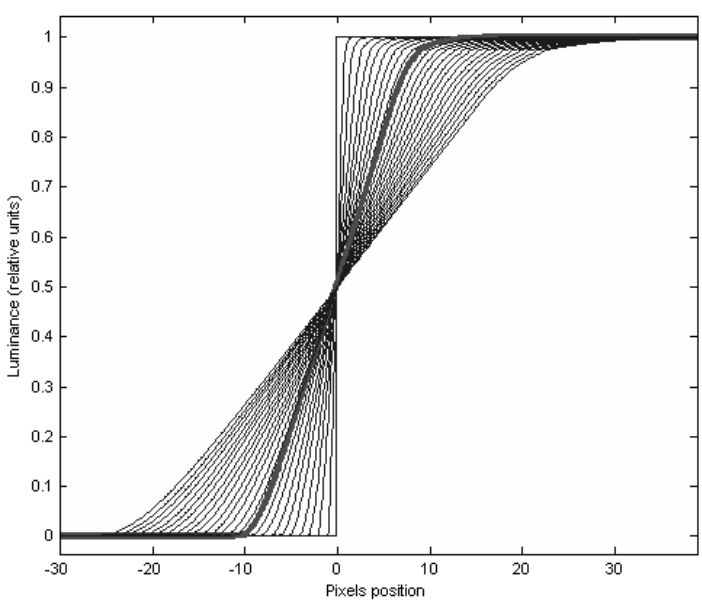

(b)

Figure 2. Example of test field (a). Stationary blurred edge profiles that can be chosen by the observers during the blur matching procedure. They are simulated by up- and down-scaling the measured blurred edge profile (thick line). 


\begin{tabular}{|c|c|c|c|c|}
\hline Id & Display type & Size & Native resolution and frame-rate & Peak luminance $\left(L_{\max }\right)$ \\
\hline \hline 1 & monitor display & $26^{\prime \prime}$ & $1920 \times 1200 @ 60 \mathrm{~Hz}$ & $300 \mathrm{~cd} / \mathrm{m}^{2}$ \\
\hline 2 & monitor display & $30 "$ & $2560 \times 1600 @ 60 \mathrm{~Hz}$ & $160 \mathrm{~cd} / \mathrm{m}^{2}$ \\
\hline 3 & raw TV panel & $37^{\prime \prime}$ & $1920 \times 1080 @ 60 \mathrm{~Hz}$ & $440 \mathrm{~cd} / \mathrm{m}^{2}$ \\
\hline
\end{tabular}

blurred edge is displayed in the upper part while a sharp edge is moving from left to right on the lower part. The method of adjustment has been used: observers have to track the moving edge in order to perceive the motion-blur and then they adjust the blur of the stationary edge until it matches their perception of the moving one. The moving edge was scrolling across the screen until observers decided to validate their adjustment, with a few-seconds break between each crossing.

The moving edge is defined by a start gray level $N_{s}$ and a final gray level $N_{f}$ (here the edge is moving from left to right, so $N_{s}$ is on the right part of the edge and $N_{f}$ on the left part). As described previously, the spatial profile of a moving edge can be obtained from the temporal step-response of the corresponding gray-to-gray transition $N_{s} \rightarrow N_{f}$. The blurred edge profiles obtained from the measurements described in the previous part have been used to set the stationary edge.

Observers have to vary the blur on the stationary edge (following the method of adjustment) until the point of subjective equality between both edges was reached. The adjustment was done by up- or down-scaling the stationary blurred edge obtained from measurements. The stationary edge could vary from a sharp edge to a very blurred edge as shown in Figure $2 \mathrm{~b}$.

Stimuli were generated with Matlab on a PC micro-computer using the PsychToolbox extension. ${ }^{11}$ Twenty gray-to-gray transitions have been tested:

$$
N_{s} \rightarrow N_{f} \mid\left\{N_{s}, N_{f}\right\} \in[0 ; 63 ; 127 ; 191 ; 255]^{2}, N_{s} \neq N_{f}
$$

\subsection{Displays under test}

Three liquid-crystal displays have been tested in this study, they are described in Table 1. Brightness and contrast parameters have been set up according to manufacturers recommendations, and a color calibration have been performed. It should be noticed that display 2 has a backlight flashing system in order to reduce motion-blur.

\subsection{Viewing conditions}

Experiments were performed in a psychophysics test room, with a surrounding luminance around $10 \mathrm{~cd} / \mathrm{m}^{2}$ and a D65 chromaticity on the walls. The screen is filled with a mean luminance $L_{\text {mean }}$ corresponding to the geometric mean of the maximum and minimum luminance of the display, $L_{\text {mean }}=\sqrt{L_{\max } L_{\min }}$. The viewing distance has been set in such a way that one pixel subtend 1.5 visual minute, for each display. This corresponds to a viewing distance of $1.87 \mathrm{H}$ for display $1,1.37 \mathrm{H}$ for display 2 , and $2.2 \mathrm{H}$ for display 3 . Whatever the display, the area where stimuli were displayed was 25-degree wide and 7.5-degree high.

\subsection{Observers}

Two labs have been involved in these experiments: IRCCyN-IVC lab (University of Nantes, France) and Acreo AB, Video and Display Quality lab (Sweden).

Displays 1 and 3 have been tested in IRCCyN-IVC. Three subjects participated to the experiments: one of the authors and two naive observers. Display 2 has been tested in Acreo. Three subjects participated to the experiments: two authors and one naive observers. All subjects possessed normal or corrected-to-normal vision (visual acuity of 0.9 or better on both eyes). They were familiar with the procedure after several training sessions, the results of which have not been considered. 


\section{RESULTS}

Figure 3 illustrates the results of the experiment, for the three displays under test, for transitions $0 \rightarrow 63$ and $63 \rightarrow 191$. On display 1 (first row), nine speeds $V$ have been tested: 4, 6, 8, 10, 12, 14, 16, 18, and 20 pixels per frame. At the viewing distance used in the experiment, that corresponds to $6,9,12,15,18,21,24,27$, and 30 degrees per second. The dashed line represents the measured blurred edge width $B E W_{m}=B E T \cdot V$ (cf. Equation 1) and dots represents the perceived blurred edge width that we write $B E W_{p}$, with the standard deviation of the subjective data represented by vertical bars. Regarding the relative stability of results on display 1 (the standard deviation of the subjective data is quite low despite of the low number of observers) we decided to test only four speeds on displays 2 and 3: 10, 15, 20, and 25 pixels per frame (i.e. 15, 22.5, 30, and 37.5 degrees per second).

It has been observed that, for each transition and for each display, the perceived blur varies linearly with the scrolling speed:

$$
B E W_{p}=k \cdot V
$$

This model is represented by the red line in Figure 3. The linear correlation coefficient between the model and the subjective data was always higher than 0.982 on the 20 transitions and 3 displays.

From Equations 1 and 2, it comes that the relationship between the perceived blurred edge width $B E W_{p}$ and the measured blurred edge width $B E W_{m}$ is linear:

$$
B E W_{p}=A \cdot B E W_{m}
$$

$A$ is the ratio between perceived motion-blur and measured motion-blur. For the majority of the tested transitions and display, this ratio is lower than 1 . This indicates that the blurred edge is actually perceived sharper than the edge obtained from objective measurements. This result may be related to motion sharpening phenomenon: ${ }^{12-14}$ blurred edges look sharper when they are moving on the retina than when stationary. ${ }^{15}$ Since, LCD motion-blur occurs because of the retina move on a stationary edge, one can assume that motion sharpening is involved here.

\section{DISCUSSION}

\subsection{Comparison with other studies}

The main result of our experiments is that the ratio $A$ between perceived and measured motion-blur is almost always lower than 1 (from 0.68 to 1.03 over all transitions and displays, with an average value of 0.91 ). This result is in accordance with that of Feng, ${ }^{10}$ but his study did not explore a lot of transitions.

Teunissen et al. have obtained some quite different results. ${ }^{9}$ They tested 8 transitions with 4 motion speeds and 17 observers on one display. Their blur matching procedure consisted, as ours, in the equalization of a static blurred edge obtained from measurements with a moving edge on which motion-blur occured. The adjustment was done by varying the speed of the moving edge: this is main difference with our experiment. For each of the 8 transitions and for the 4 tested speeds, their results showed that the adjusted speed was very close to the speed used to simulate the still blurred edge. In other terms, the ratio between perceived and measured motion-blur was equal to 1 for all transitions. Moreover, observers were asked to rate the match between both blurred edges with a five-grade quality scale and the average score were above 4.4 in all cases, indicating a very good match.

The difference of tasks between our two experiments may explain the different results, as well as the fact that, in their experiment Teunissen et al. used a moving block instead of a moving edge in our experiment. As a consequence, for a block with a gray level $N_{i}$ moving on a background with a gray level $N_{j}$, the blur matching procedure was done on both transitions $N_{i} \rightarrow N_{j}$ and $N_{j} \rightarrow N_{i}$, whereas in our experiment only one transition was tested at a time. The equalization of both rising and falling edges at the same time may have introduced a bias. 


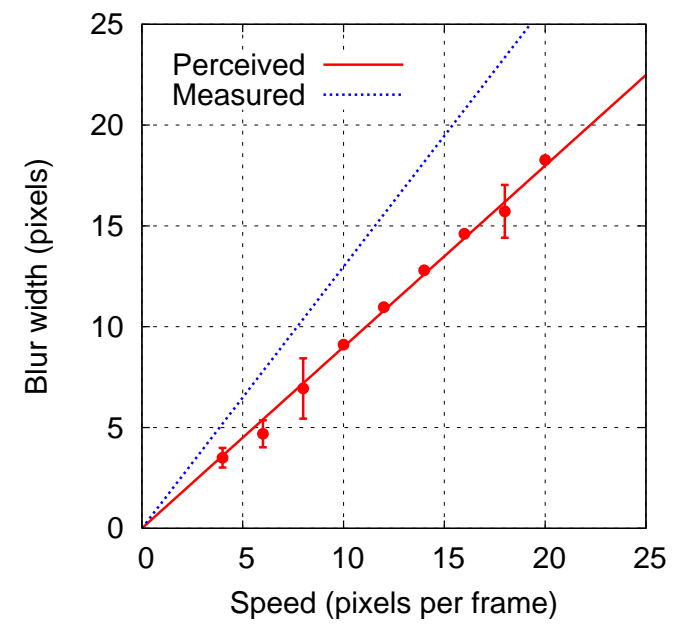

(a) $0 \rightarrow 63$ on display 1 .

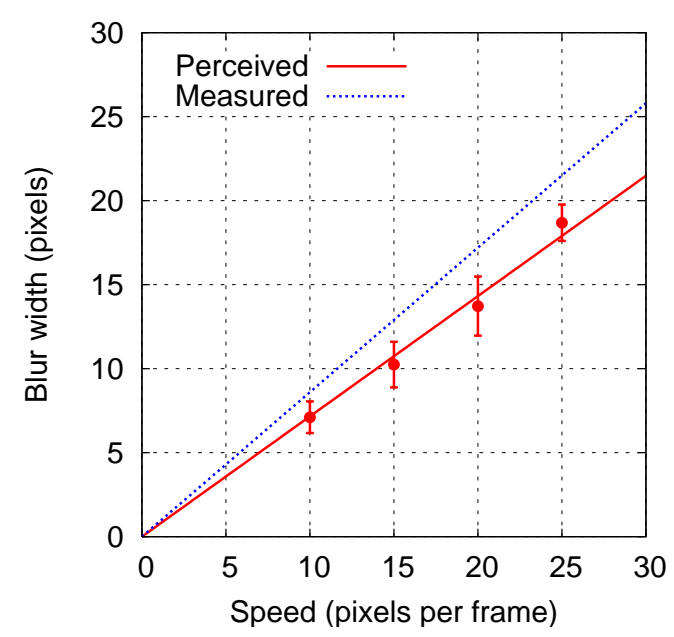

(c) $0 \rightarrow 63$ on display 2 .

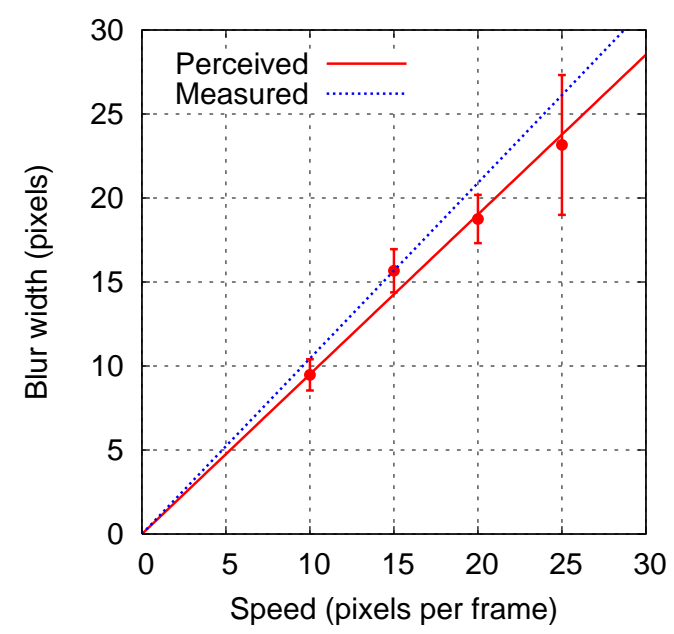

(e) $0 \rightarrow 63$ on display 3 .

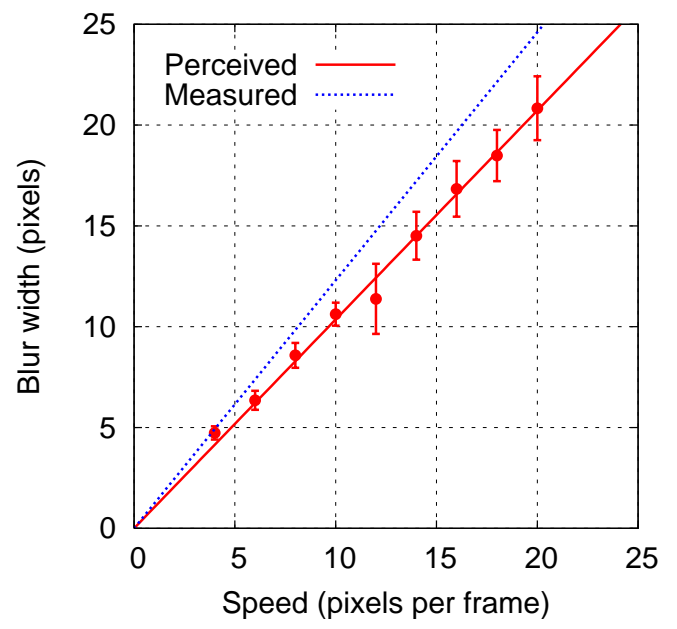

(b) $63 \rightarrow 191$ on display 1 .

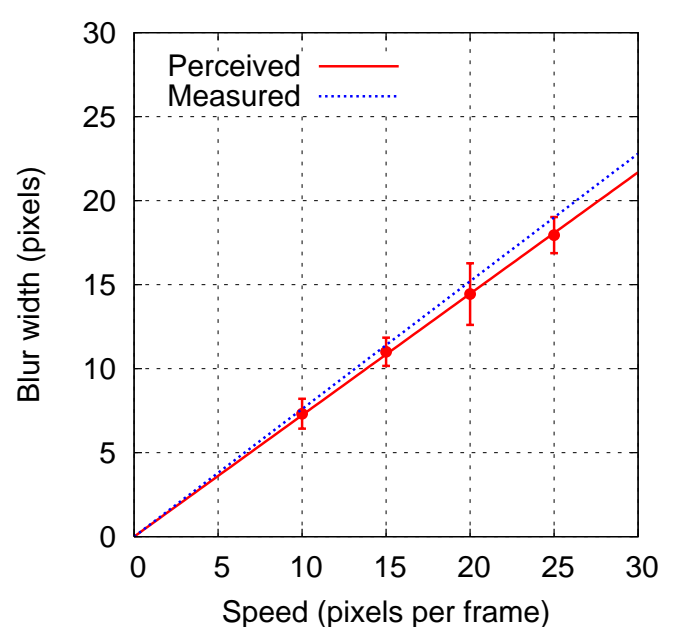

(d) $63 \rightarrow 191$ on display 2 .

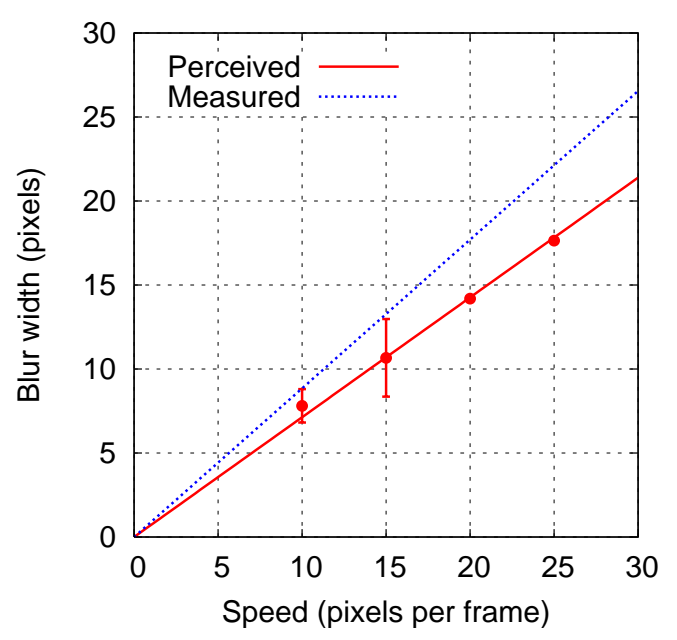

(f) $63 \rightarrow 191$ on display 3 .

Figure 3. Measured motion-blur (dashed line) and perceived motion-blur (dots) as a function of speed. Transitions $0 \rightarrow 63$ (left) and $63 \rightarrow 191$ (right), on display 1 (first row), display 2 (second row) and display 3 (third row). Vertical bars represent the standard deviation of the subjective data. 

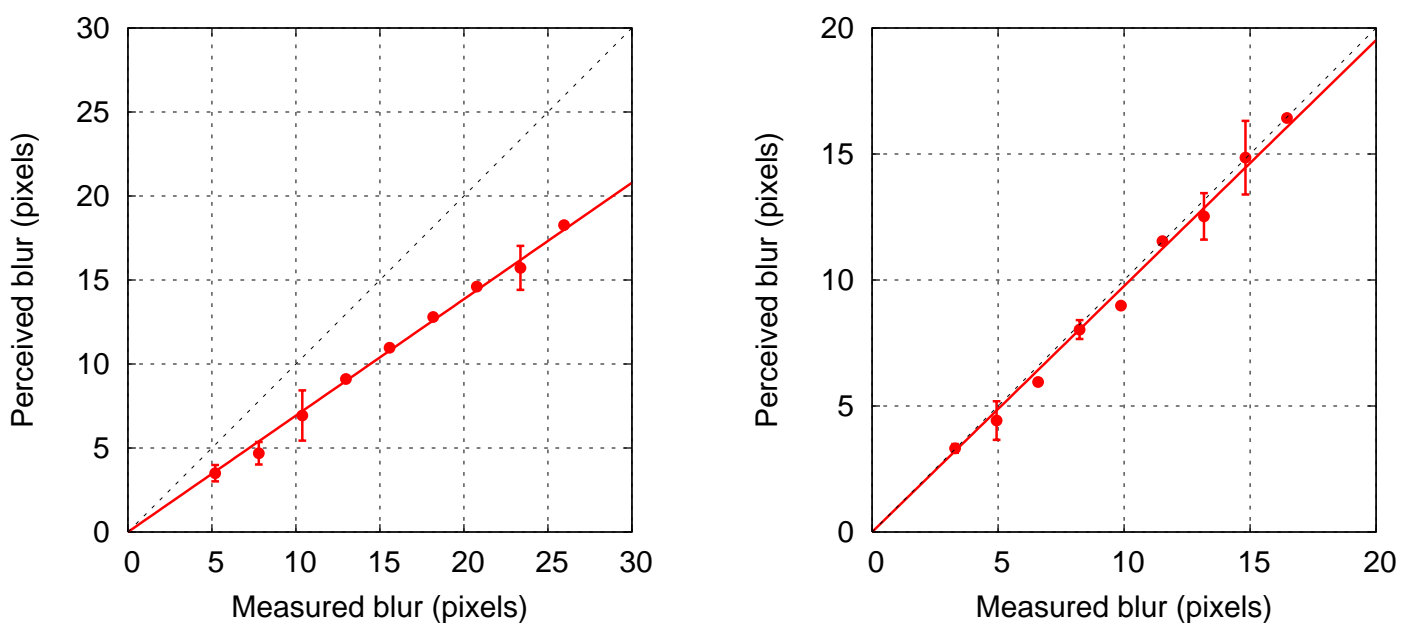

Figure 4. Perceived motion-blur as a function of measured motion-blur, for transitions $0 \rightarrow 63$ (left) and $0 \rightarrow 255$ (right), for display 1. Vertical bars represent the standard deviation of the subjective data. The red line represents the model $B E W_{p}=A \cdot B E W_{m}$, with $A=0.69$ for $0 \rightarrow 63$ (left) and $A=0.98$ for $0 \rightarrow 255$ (right).

\subsection{Evolution of ratio $A$ as a function of transitions}

It has been observed that the ratio $A$ between perceived motion-blur and measured motion-blur is lower for edges with low dynamic and tends towards 1 as the dynamic of the edge is increasing. For example, Figure 4 presents the perceived motion-blur as a function of measured motion-blur for transitions $0 \rightarrow 63$ and $0 \rightarrow 255$ on display 1. The red line is fitted to subjective data according to Equation 2. The ratio $A$ is the slope of the line: 0.69 for transition $0 \rightarrow 63$ and 0.98 for transition $0 \rightarrow 255$.

On Figure 5, the ratio $A$ between perceived and measured motion-blur is plotted as a function of the edge dynamic, for displays 1 and 2 . The edge dynamic is defined as the absolute difference between the start luminance $L_{s}$ and the final luminance $L_{f}$. It can be observed that $A$ is increasing as the edge dynamic increases. The relationship is slightly different according to the type of transitions. For rising transitions $\left(L_{s}<L_{f}\right)$, linear correlation coefficients are quite reliable $(0.868$ and 0.836$)$, but for falling transitions $\left(L_{f}<L_{s}\right)$, the growing of the relationship is less obvious and the linear correlation coefficients are not so relevant (less than 0.7).

Figure 6 presents the same results as Figure 5.a but here the transitions are gathered regarding the value of the start gray level $N_{s}$. One can see that for a given start gray level $N_{s}$, the evolution of the ratio $A$ is increasing rather linearly as $N_{f}$ increases. Of course, more results are needed to draw sound conclusions, but it is another evidence for the fact that ratio $A$ is decreasing with contrast.

The study of the ratio $A$ on display 3 did not permit to bring out a particular tendency. This may be due to the fact that display 3 presented some important overshoots on its temporal step-responses, and so on the blurred edge profile, due to overdriving. As a consequence, the matching between simulated and perceived edges was more difficult to obtain.

\section{CONCLUSION}

The psychophysical study of LCD motion-blur described in this work has shown that objective motion-blur obtained from physical measurements and perceived motion-blur are highly correlated. However, perceived blur is lower than physical blur for almost all cases. This result may be related to the motion sharpening phenomenon. 


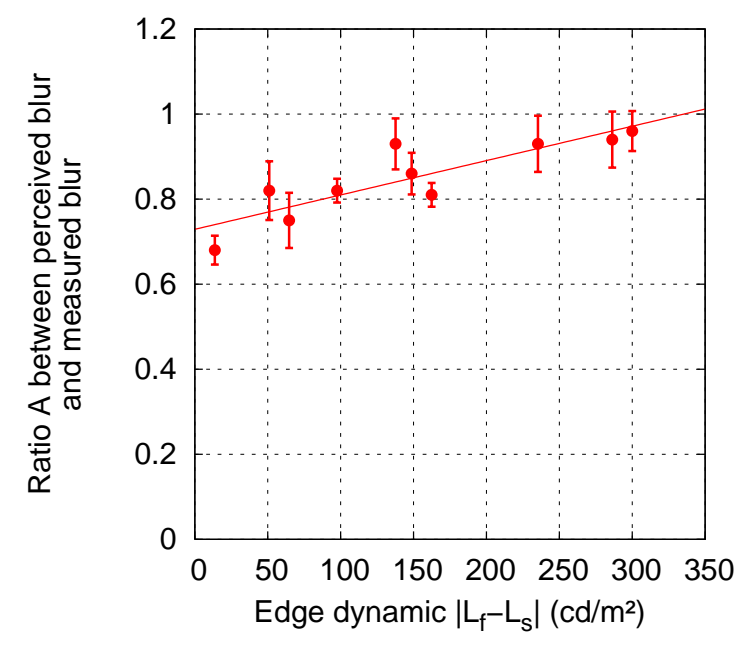

(a) Display 1 - Rising transitions.



(c) Display 2 - Rising transitions.

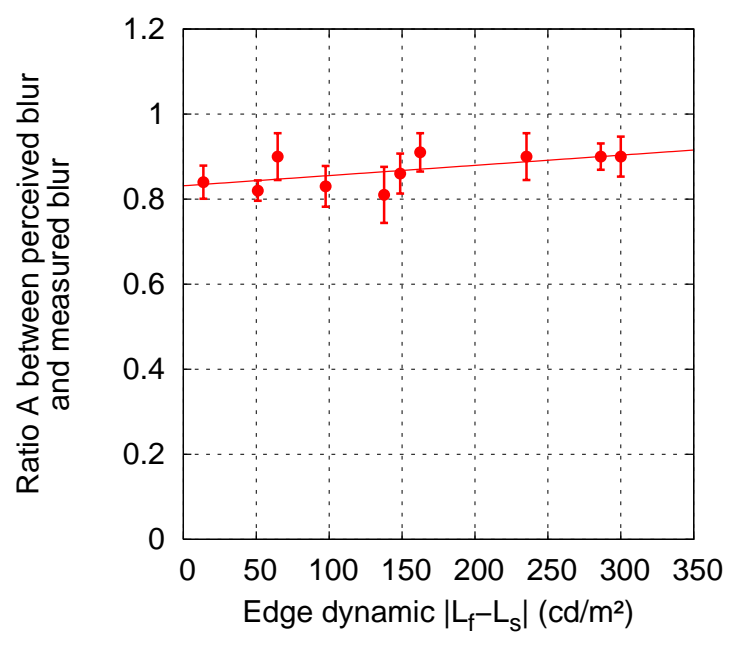

(b) Display 1 - Falling transitions.

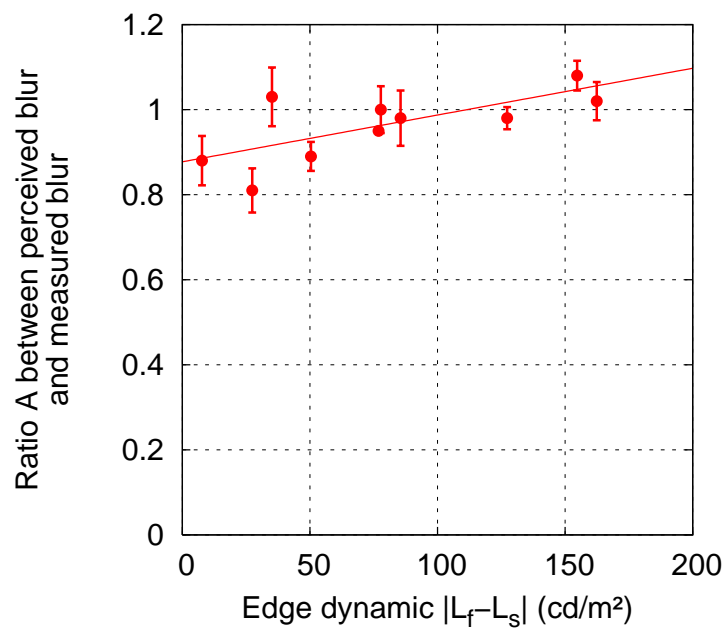

(d) Display 2 - Falling transitions.

Figure 5. Ratio $A$ between perceived and measured motion-blur as a function of the edge dynamic. For rising transitions on display 1 (a): $C C=0.868$. For falling transitions on display 1 (b): $C C=0.679$. For rising transitions on display 2 (c): $C C=0.836$. For falling transitions on display $2(\mathrm{~d}): C C=0.693$. 


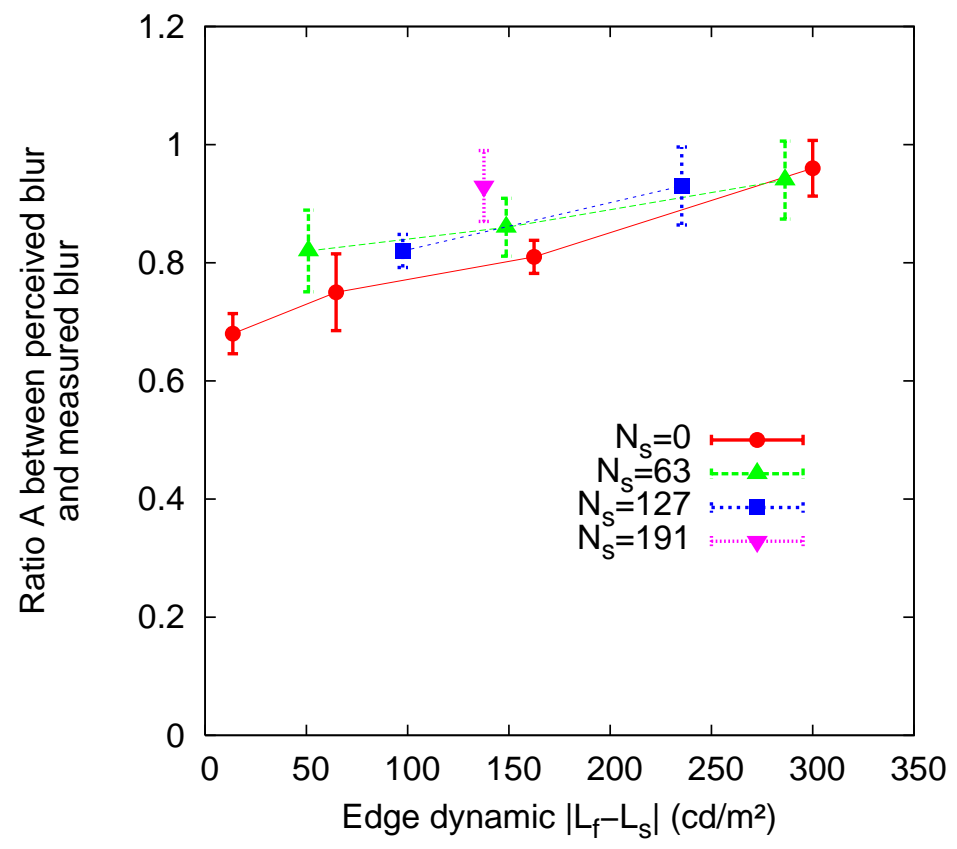

Figure 6. Ratio $A$ between perceived and measured motion-blur as a function of the edge dynamic, for rising transitions on display 1. Idem as Figure 5.a but transitions are linked regarding the start gray level $N_{s}$.

Results show that the ratio between perceived blur and measured blur is close to 1 for high contrasted transitions and is decreasing as the dynamic of the edge descreases. This indicates that sharpening is stronger for low contrasted edges. This effect is particularly visible for rising transitions, less for falling transitions.

Current VESA recommendation about motion-blur measurements ${ }^{16}$ suggest to take the average of motionblur measurements over transitions as a motion-blur value for the display. Results of this work tend to prove that perception of LCD motion-blur is not constant with transitions and that measurements should be weighted as a function of contrast to obtain a "perceptual" motion-blur score.

\section{REFERENCES}

1. T. Kurita, "Moving picture quality improvement for hold-type AM-LCDs," in SID Symposium Digest of Technical Papers, 32, pp. 986-989, SID, June 2001.

2. K. Oka and Y. Enami, "Moving picture response time (MPRT) measurement system," in SID Symposium Digest of Technical Papers, 35, pp. 1266-1269, SID, May 2004.

3. X. fan Feng, H. Pan, and S. Daly, "Comparison of motion blur measurement in LCD," in SID Symposium Digest of Technical Papers, 38, pp. 1126-1129, SID, May 2007.

4. S. Tourancheau, K. Brunnström, B. Andrén, and P. Le Callet, "LCD motion-blur estimation using different measurement methods," Journal of the Society for Information Display 17(3), 2009.

5. H. Pan, X.-F. Feng, and S. Daly, "LCD motion blur modeling and analysis," in IEEE International Conference on Image Processing, 2005. ICIP 2005., 2, pp. 21-24, September 2005.

6. A. B. Watson, "The Spatial Standard Observer: A human vision model for display inspection," in SID Symposium Digest of Technical Papers, 37, pp. 1312-1315, SID, June 2006.

7. S. Tourancheau, K. Brunnström, B. Andrén, and P. Le Callet, "Motion blur estimation on LCDs," in SID Symposium Digest of Technical Papers, 39, SID, May 2008.

8. J. Someya, "Correlation between perceived motion blur and MPRT measurement," in SID Symposium Digest of Technical Papers, 36(1), pp. 1018-1021, SID, 2005. 
9. K. Teunissen, Y. Zhang, X. Li, and I. Heynderickx, "Method for predicting motion artifacts in matrix displays," Journal of the Society for Information Display 14(10), pp. 957-964, 2006.

10. X.-F. Feng, "LCD motion-blur analysis, perception, and reduction using synchronized backlight flashing," in Proceedings of the SPIE Conf. Human Vision and Electronic Imaging XI, 6057, Electronic Imaging 2006, Janvier 2006.

11. D. H. Brainard, "The Psychophysics Toolbox," Spatial Vision 10(4), pp. 433-436, 1997.

12. P. J. Bex, G. K. Edgar, and A. T. Smith, "Sharpening of drifting, blurred images," Vision Research 35, pp. 2539-2546, 1995.

13. S. T. Hammett and P. J. Bex, "Motion sharpening: evidence for the addition of high spatial frequencies to the effective neural image," Vision Research 36, pp. 2729-2733, 1996.

14. T. Takeuchi and K. K. De Valois, "Sharpening image motion based on the spatio-temporal characteristics of human vision," in Proceedings of the SPIE Conf. Human Vision and Electronic Imaging X, 5666, 2005.

15. M. A. Georgeson and S. T. Hammett, "Seeing blur: 'motion sharpening' without motion," Proc. R. Soc. Lond. B 269, pp. 1429-1434, 2002.

16. VESA, "Flat Panel Display Measurements (FPDM2)," Tech. Rep. 2.0 Update, Video Electronics Standards Association, May 2005. 